\title{
On Strong Correlations between Timing Irregularities and Surface Magnetic Field of Rotation-Powered Pulsars
}

\author{
Evaristus Uzochukwu Iyida1,2*, Christian Ikechukwu Eze1,2, Innocent Okwudili Eya ${ }^{1,3}$ \\ ${ }^{1}$ Astronomy and Astrophysics Research Group, Department of Physics and Astronomy, University of Nigeria, Nsukka, Nigeria \\ ${ }^{2}$ Department of Physics and Astronomy, Faculty of Physical Sciences, University of Nigeria, Nsukka, Nigeria \\ ${ }^{3}$ Department of Science Laboratory Technology, Faculty of Physical Sciences, University of Nigeria, Nsukka, Nigeria \\ Email: *uzochukwu.iyida@unn.edu.ng
}

How to cite this paper: Iyida, E.U., Eze, C.I. and Eya, I.O. (2020) On Strong Correlations between Timing Irregularities and Surface Magnetic Field of Rotation-Powered Pulsars. International Journal of Astronomy and Astrophysics, 10, 346-355.

https://doi.org/10.4236/ijaa.2020.104019

Received: April 6, 2020

Accepted: December 25, 2020

Published: December 28, 2020

Copyright $\odot 2020$ by author(s) and Scientific Research Publishing Inc. This work is licensed under the Creative Commons Attribution International License (CC BY 4.0).

http://creativecommons.org/licenses/by/4.0/

\begin{abstract}
Using a large homogenous sample of Jodrell Bank Observatory (JBO) rotation-powered pulsars with derived pulsar parameter obtained from the Australian Telescope National Facility (ATNF) catalogue, we investigated the degree of statistical dependence of timing noise on surface magnetic field for our sample. The distributions of the timing irregularities parameters reveal a striking trend, in which pulsars with large timing noise parameters on average, have large surface magnetic field with up to 5 orders of magnitude. Regression analysis of the data reveals significant correlations $(r \geq 0.80)$ between timing noise parameters and the surface magnetic field. The implications of the observed significant relationships are discussed.
\end{abstract}

\section{Keywords}

Methods: Statistical, Stars: Neutron, Pulsars: General

\section{Introduction}

Highly magnetized, fast rotating neutron stars called pulsars are one of the most stable natural rotators in the universe. Their clock-like behavior has a widespread range of applications from detections of the stochastic background of gravitational waves [1] [2] to testing theories of gravity [3] [4]. However, the clocks are far from perfect as they slow down with time leading to increase in spin frequency $v$ and its first time derivative $\dot{v}$ according to the spin down law [5] given as

$$
\dot{v}=-K v^{n}
$$


or its equivalent in terms of the spin period and its first time derivative given as

$$
\dot{P}=K P^{2-n}
$$

where the exponent $n$ is the braking index and $K$ is a positive constant based on standard spin-down model of a dipole rotator in a vacuum. The surface magnetic field can be explicitly expressed [6] as

$$
B_{\text {surf }}=\sqrt{\frac{K 6 c^{2} I}{R^{6} \sin \alpha}}
$$

where $I$ and $R$ are respectively, the moment of inertia and the radius; $\alpha$ is the angle between the magnetic and the spin axes of pulsar and $c$ is the speed of light. On the other hand, direct measurements of pulsar braking index $n$ can be accomplished through measurements of pulse spin period and its first and second time derivatives respectively and is given [5] [7] as

$$
n=2-\frac{P \ddot{P}}{\dot{P}^{2}}
$$

where $\ddot{P}$ is period second time derivative. Conversely, the radiations emitted by pulsars are at the expense of another physical quantity, which can be inherent to the pulsar or external. Those pulsars whose emissions are at the expense of the rotational kinetic energy are known as rotation-powered pulsars [5]. For these pulsars (radius of $10 \mathrm{~km}$ and moment of inertia $\sim 10^{45} \mathrm{~g} \cdot \mathrm{cm}^{-2}$ ), the estimate of the surface magnetic field is given [6] as

$$
B_{\text {surf }}=3.2 \times 10^{19} \sqrt{P \dot{P}}(\text { Gauss })
$$

The detailed long-term timing observations of these pulsars have revealed that the otherwise smooth spindown of most radio pulsars is prone to a wide variety of disruptions called timing noise, which have proven extremely useful in the use of pulsars as physical tools. At present, the observed timing activities are broadly grouped into glitches and timing noise. Glitches are sudden tiny jumps in pulsar spin rate which are usually accompanied by increase in the magnitude of frequency derivative [8]. Glitches are uncommon spectacular events characterized by recovery of the jumps in frequency and its first time derivatives on a large range of timescales [9] [10]. Currently, it is generally agreed that glitches come from various complex dynamical changes within the interior of neutron star; hence, the study could offer valuable information about the internal structure and dynamics of neutron stars [11] [12] [13]. On the contrary, timing noise, is the random, sustained fluctuations in the pulsars' spin parameters, that lasts from days to years and results in excess timing phase residuals [14]. It is believed to occur often in rotation-powered pulsars in which the pulse arrival times wander continuously about the fitted ephemeris [15] [16]. Even though the predominance of timing noise (as unmodeled structures in pulsar timing residuals in excess of what is expected from measurement error) within the pulsar samples seems not fully established [17] [18] [19], our understanding of the phenomenon is still largely poor [7] [19]. Meanwhile, timing noise has been attributed to some 
form of variations in the coupling between neutron star crust and its superfluid core [20] [21] and/or torque fluctuations in the magnetosphere [22] [23] [24] [25].

Hitherto, a number of parameters were introduced to illustrate and measure the amount of timing noise in pulsars. Theoretically, a qualitative measurement of the level of timing noise activity in pulsars can be obtained from the surplus phase residuals remaining after accounting for its deterministic spin-down [21] [26]. [27] in that effect, fitted the observed rotational phase of each pulsar with a function form

$$
\varphi_{(t)}=\varphi_{0}+\left(v_{t-t_{0}}\right)+\frac{1}{2} \dot{v}\left(t-t_{0}\right)^{2}+\frac{1}{2} \ddot{v}\left(t-t_{0}\right)^{3}+\cdots,
$$

which is a Taylor series expansion around a reference time $t_{0}$, where the terms $v, \dot{v}$, and $\ddot{v}$ are the spin frequency, its first and second time derivatives respectively, while $\varphi_{(t)}$ is the phase at a time $t$. The activity parameter is the logarithm of the ratio of the root-mean-square deviation over a $12 \mathrm{~ms}$ observation for a given pulsar to the same quantity in the crab pulsar [15], given as

$$
A=\log \left[\frac{\sigma_{T N}(m, T)}{\sigma_{T N}(m, T)_{c r a b}}\right]
$$

where $m$ is the order of the polynomial fit to the data, $T$ is the time span of the fit in days, $\sigma_{T N}(m, T)$ is the timing noise contribution to the gross root mean square phase residuals obtained from the quadrature difference between the mean squares of the observed phase residuals and the measurement errors [16] while $\sigma_{T N}(m, T)_{c r a b}$ is the timing noise of the crab pulsar (in ms). [27] introduced the stability parameter expressly used to characterize the intensity of the fluctuations observed in pulsars, which is based on the amplitude of the observed spin frequency second time derivative and can be written as

$$
\Delta_{8}=\log \left(\frac{1}{6}\left|\ddot{v}_{\text {noise }}\right|\left(10^{8} s\right)^{3}\right)
$$

where the $\ddot{v}$ is frequency second time derivative over a period of $10^{8}$. The cubic term dominates the variance for a timing noise in the group of average of any red process with a monotonically decaying spectrum. Pulsar clock stability parameter $\sigma_{Z}(\tau)$ introduced by [28] is a dimensionless Allan variance-like parameter used to quantify the degree of rotational stability in rotation-powered pulsars and is defined as

$$
\sigma_{Z}(T)=\frac{1}{2 \sqrt{5}}\left[\frac{\sigma_{\ddot{v}}(T)}{v}\right] T^{2},
$$

where $\sigma_{\ddot{v}}$ is the root-mean-square of $\ddot{v}$, measured over observing time span $T$ [14]. Conventionally, $T=10$ yrs. Meanwhile, a direct quantification of the contamination in $\ddot{v}$ from the standard timing activity by all forms of timing fluctuation is [29]

$$
\sigma_{R 23}(T)=\sqrt{\sigma_{R}^{2}(2, T)-\sigma_{R}^{2}(3, T)},
$$


where $\sigma_{R}^{2}(2, T)$ and $\sigma_{R}^{2}(3, T)$ are, respectively, the variances of the timing residuals over $T$ after a second and third-order polynomial fits. The developments of timing noise parameters with various spin-down properties have been used for detailed statistical study of timing noise in pulsar populations [14] [30] [31] [32] [33]. Specifically, some statistical analyses of timing noise phenomenon in rotation-powered pulsars involving spin-down properties have shown that timing noise is common among young pulsars [14] [18] [31] [34] [35] [36]. However, no result of timing observations has revealed that timing irregularities are strongly dominated by the observed surface magnetic field of a sizeable number of pulsars over long timescales ( $>10$ years). Thus, the main idea behind this paper is to explore the contributions of the pulsar's surface magnetic field strength on timing noise irregularities and the intrinsic evolution of pulsars.

\section{Data Analysis and Results}

The data used in the current analysis is a large homogeneous sample of Jodrell Bank Observatory (JBO) radio pulsars with significant measurements of timing noise parameters estimated from radio timing data with time span length of over four decades. The activity parameter was computed using Equation (7) and taking parameters from [14] on the assumption that the method used effectively whitened the timing residuals of the pulsars in the sample. A sample of $366 \mathrm{ob}-$ jects with complete information on rotation-powered pulsars' stability parameter $\left(\Delta_{8}\right)$ and pulsar clock stability parameter $\sigma_{z}(\tau)$ were compiled from [14]. Also, the timing noise statistic $\sigma_{23}$ was calculated from the information in [14] using Equation (10). The corresponding surface magnetic field was extracted from the Australian Telescope National Facility (ATNF) Pulsar website available at the website http://www.atnf.csiro.au/research/pulsar/psrcat. We used both analytic and statistical methods for this study. The statistical method was used to estimate the measures of dispersion of our sample while the correlation analysis was used to check for any form of relationship between the parameters. The Pearson product moment enabled us to calculate the correlation coefficient $r$, which provides the strength of correlation between the parameters. The distributions of the activity parameter and pulsar clock stability parameter are shown in Figure 1(a) and Figure 1(b). For the activity parameter, it ranges from -2.24 to 1.83 with mean value value of $\sim-0.83 \pm 0.02$, and median value of $\sim-0.46$. The figure shows that about $\sim 210(\sim 81 \%)$ of the pulsars in the sample have activity parameter less than zero. However, in the case of Pulsar clock stability parameter, the values are from -13.13 to -7.04 with mean value of $\sim-10.81 \pm 0.02$ and median value of -10.09 with few $\sim 112$ (constituting $23 \%$ of pulsars) have clock stability parameter values less than the mean value. Figure $1(\mathrm{c})$ and Figure 1 (d) are the distributions of stability parameter and timing noise statistic $\sigma_{23}$. The stability parameter ranges from -2.50 to -0.92 with mean and median values of $\sim-1.38 \pm 0.03$ and $\sim-1.02$ respectively in a multimodal configuration. For the timing noise statistic, it ranges from -1.08 to 3.50 with mean value of 

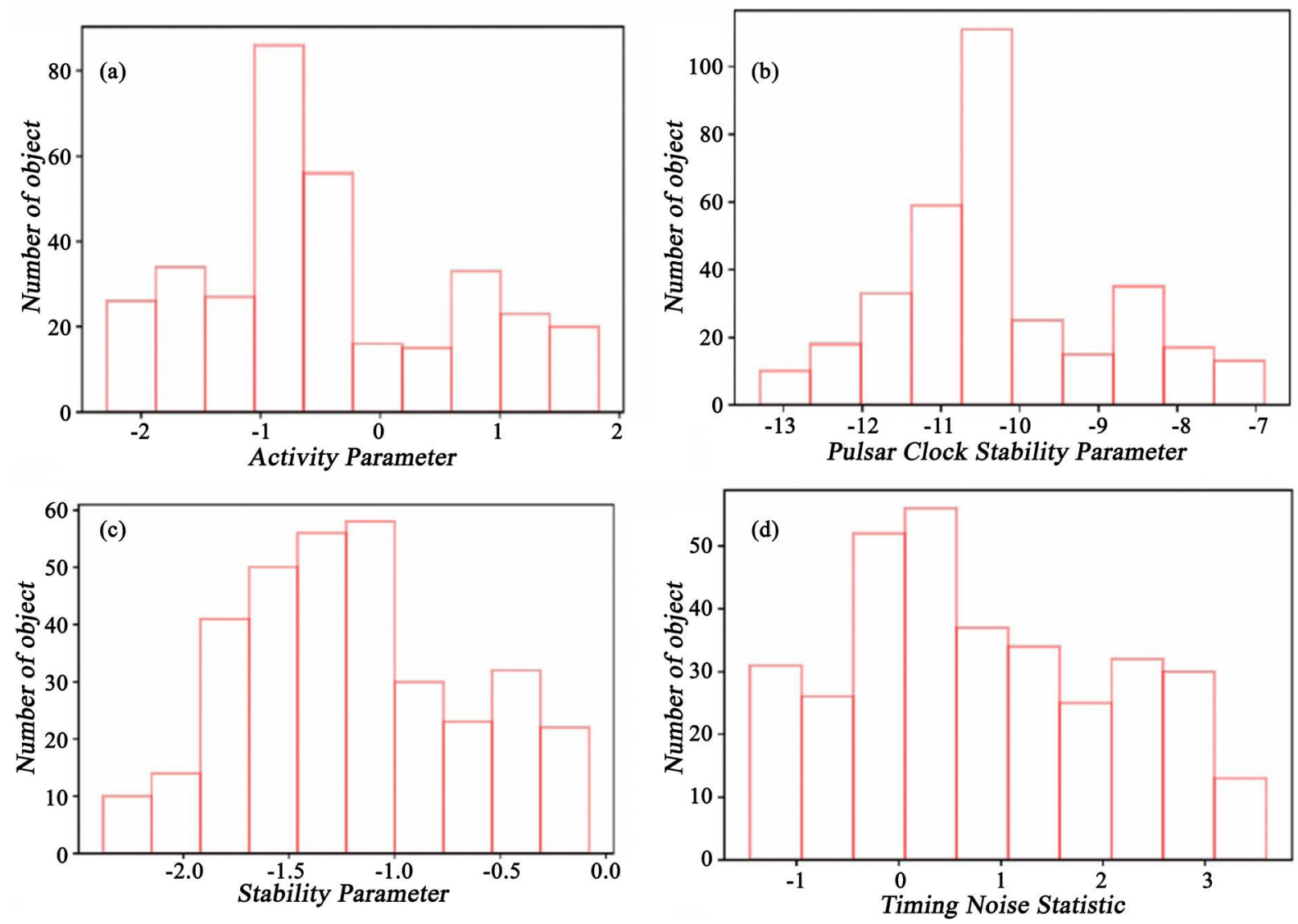

Figure 1. Histograms showing distributions of (a) Activity parameter (b) pulsar clock stability parameter (c) stability parameter (d) Timing Noise statistic of our sample.

$\sim 1.27 \pm 0.02$ and median value of 1.01 . The distribution appears to be bimodal, which could suggest two kinds of timing noise statistics, and skews to the right (with skewness $(\mu)$ in the range: $0.03 \leq \mu \leq 0.02)$ with many pulsars $(\sim 78 \%)$ having values less than the mean value. In order to evaluate for any possible relationship between timing noise parameters and surface magnetic field, we made plots of timing noise parameters as a function of surface magnetic field.

In doing this, a logarithmic value of surface magnetic field was used to reduce the nearness in the value. Figure 2(a) \& Figure 2(b) show the bivariate scatter plots of the activity parameter and pulsar clock stability parameter against the surface magnetic field for the pulsars in our sample. In the figures, the magnetic field of pulsar population in our sample is in the range $1 \times 10^{7} \leq B \leq 1.58 \times 10^{14}$ Gauss. The figures clearly show an upward sloping trend with closely clustered scatter points depicting that pulsars with large values of both activity parameter and pulsar clock stability parameter have large values of surface magnetic field. A linear regression analysis of our data yields a positive correlation coefficient $(r$ $\sim 0.88$ ) for the two variables being studied. The exact form of their relationship is

$$
\begin{aligned}
& A=(0.70 \pm 0.30) B_{\text {surf }}+(-2.40 \pm 0.10) \\
& \sigma_{z}=(0.61 \pm 0.20) B_{\text {surf }}+(-13.3 \pm 0.20)
\end{aligned}
$$



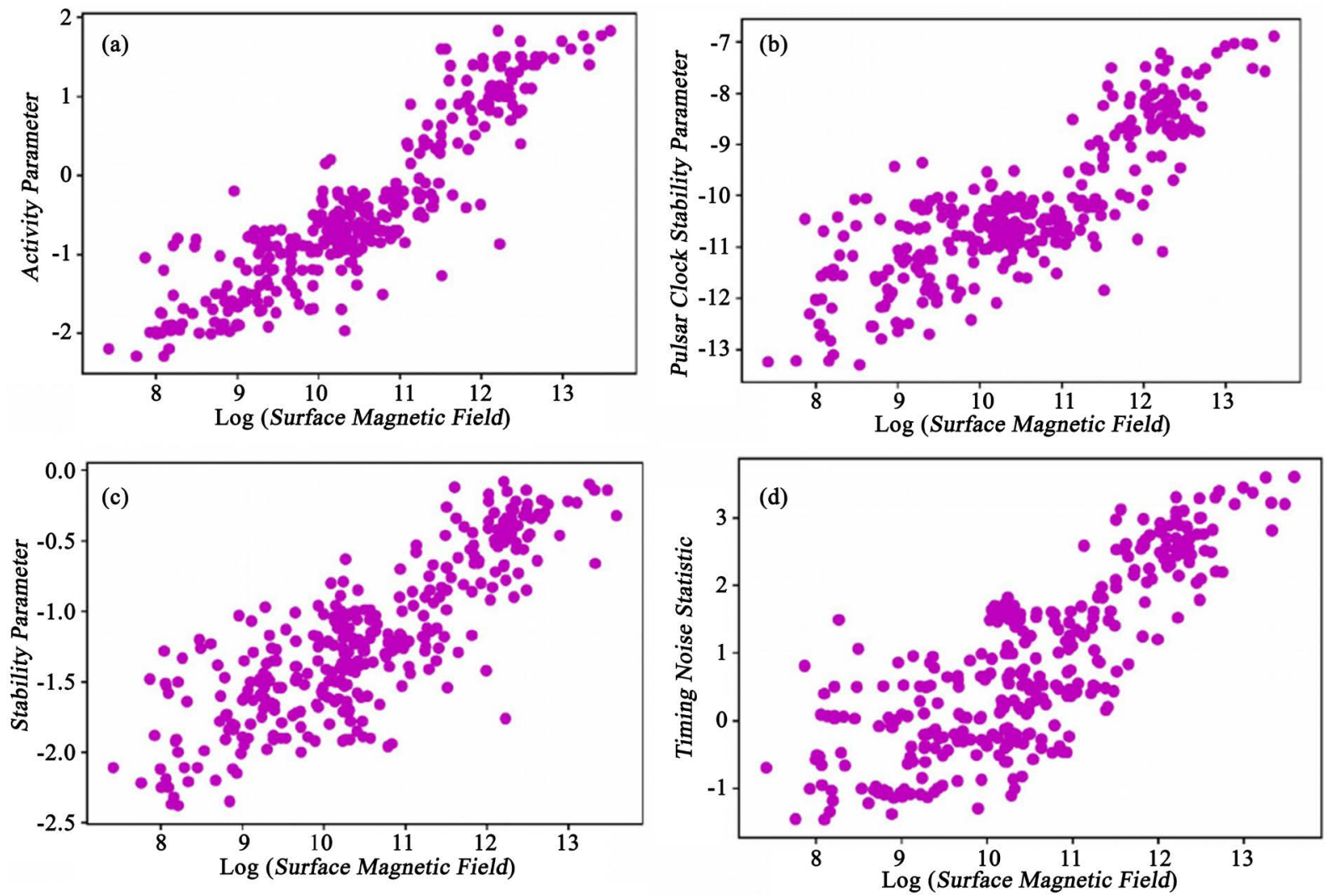

Figure 2. Scatter plots of (a) activity parameter (b) pulsar clock stability parameter (c) stability parameter (d) timing noise statistic against the logarithm of surface magnetic field.

However, Figure 2(c) \& Figure 2(d) show the scatter plots of the stability parameter and timing noise statistic $\sigma_{23}$ against the surface magnetic field. The plots, which show an upward sloping tendency indicates that pulsars with large surface magnetic field exhibit high timing noise irregularities. A regression analyses of our data show that the stability parameter and timing noise statistic $\sigma_{23}$ have significant positive correlation with the surface magnetic field $(r \sim>0.80)$ in each case with the linear relationship of the form

$$
\begin{aligned}
& \Delta_{8}=(0.78 \pm 0.20) B_{\text {surf }}+(-2.43 \pm 0.10) \\
& \sigma_{23}=(0.97 \pm 0.20) B_{\text {surf }}+(-2.10 \pm 0.10)
\end{aligned}
$$

implying that surface magnetic is a key promoter of timing noise irregularity in these pulsars.

\section{Discussion}

The result of the statistical analysis of a large data base of rotation powered pulsars with significant measurements of timing noise parameters and their effects on the intrinsic surface magnetic field has been presented. The surface magnetic field is a derived and rotational parameter and equally an intrinsic property of radio pulsar. The radio pulsars' spin period and its first time derivative is a key property of radio pulsars [7]. The surface magnetic field is dependent on the 
pulsar spin period and its first time derivative, thus, the existence of a positive relationship between the pulsar spin periods and its first time derivative on the timing noise of pulsars is remarkable [33]. Hence, the surface magnetic field could be observed to show positive relationship with the timing noise parameters. This implies that timing noise is widespread and prevalent among radio pulsars that have large surface magnetic field. We observed that pulsars with high values of timing noise parameters are associated with large surface magnetic field. Previous results show that young pulsars $\left(10^{4}\right.$ years $)$ appear to show more timing noise activity than their old, more stable counterparts [14] [17] [27] [33] [37] [38]. Consequently, the existence of this dependence of timing noise activity on the surface magnetic field is prominent among young pulsars with large surface magnetic field. A significant feature in the plots of the timing noise parameters against the surface magnetic field with the values of the surface magnetic field in the of $10^{11}-10^{12}$ Gauss is remarkable in that this range corresponds to the triple pulsar system PSR 1714-34 in the globular system. These high values of surface magnetic field with the timing noise parameters could be attributed to the acceleration of pulsars in the globular cluster due to their strong gravitational field. It is believed that timing noise is a direct consequence of the source of the intrinsic strong gravitation and fluctuations in the globular system. Moreover, the very strong $(r \geq 0.80)$ correlations which characterize the relationships between timing noise parameters and the surface magnetic field is a clear suggestion that pulsars with high surface magnetic fields on the average display more timing noise than their counterparts.

\section{Conclusion}

The observed surface magnetic field of the radio pulsars has been carefully studied for characterization with the pulsar timing noise. Our results showed that the strength of radio pulsar surface magnetic field have obvious significant effects on the timing noise parameters. There is existence of positive relationship between the parameters that characterize timing noise and the radio pulsar surface magnetic field. This is a clear indication that pulsars with large surface magnetic field are highly susceptible to timing noise activity.

\section{Conflicts of Interest}

The authors declare no conflicts of interest regarding the publication of this paper.

\section{References}

[1] Shannon, R.M., Ravi, V., Lentati, L.T., Lasky, P.D., Hobbs, G., Kerr, M., et al. (2015) Gravitational Waves from Binary Supermassive Blackhole Missing in Pulsar Observations. Science, 349, 1522-1525. https://doi.org/10.1126/science.aab1910

[2] Arzoumanian, Z., Baker, P.T., Brazier, A., Burke-Spolaor, S., Chamberlin, S.J., Chatterjee, S., et al. (2018) The NANOGrav 11 Year Dataset: Pulsar Timing Constraints on the Stochastic Gravitational Wave Background. Astrophysical Journal, 
859, 47. https://doi.org/10.3847/1538-4357/aabd3b

[3] Kramer, M., Stairs, I.H., Manchester, R.N., McLaughlin, M.A., Lyne, A.G., Ferdman, R.D., et al. (2006) Tests of General Relativity from Timing the Double Pulsar. Science, 314, 97-102. https://doi.org/10.1126/science.1132305

[4] Cameron, A.D., Champion, D.J., Kramer, M., Bailes, M., Barr, E.D., Bassa, C.G., et al. (2018) The High Timing Resolution Universe Pulsar Survey-XIII. PSR J1757-1854, the Most Accelerated Binary Pulsar. Monthly Notices of the Royal Astronomical Society: Letters, 475, L57-L61. https://doi.org/10.1093/mnrasl/sly003

[5] Manchester, R.N. and Taylor, J.H. (1977) Pulsars. W.H. Freeman and Company, San Francisco.

[6] Allen, M.P. and Horvath, J.E. (1997) Glitches, Torque Evolution and the Dynamics of Young Pulsars. Monthly Notices of the Royal Astronomical Society, 287, 615-621. https://doi.org/10.1093/mnras/287.3.615

[7] Lorimer, D.R. and Kramar, M. (2005) Handbook of Pulsar Astronomy. Cambridge University Press, Cambridge.

[8] Yu, M., Manchester, R.N., Hobbs, G., Johnston, S., Kaspi, V.M., Keith, M., et al. (2013) Detection of 107 Glitches in 36 Southern Pulsars. Monthly Notices of the Royal Astronomical Society, 429, 688-724. https://doi.org/10.1093/mnras/sts366

[9] Flanagan, C.S. (1990) A Brief History of Pulsar Time. Nature, 345, 416-417. https://doi.org/10.1038/345416a0

[10] Urama, J.O. (2002) Glitch Monitoring in PSR B1046-58 and B1737-30. Monthly Notices of the Royal Astronomical Society, 330, 58-62. https://doi.org/10.1046/j.1365-8711.2002.05099.x

[11] Flanagan, C.S. (1990) Unpublished PhD Thesis, University of Grahamstown, South Africa.

[12] Melatos, A., Peralta, C. and Wyithe, J.S.B. (2008) Avalanche Dynamics of Radio Pulsar Glitches. Astrophysical Journal, 672, 1103-1118.

https://doi.org/10.1086/523349

[13] Eya, I.O., Urama, J.O. and Chukwude, A.E. (2017) Angulsar Momentum Transfer and Fractional Moment of Inertia in Pulsar Glitches. Astrophysical Journal, 840, 56. https://doi.org/10.3847/1538-4357/aa6b55

[14] Hobbs, G., Lyne, A.G. and Kramar, M. (2010) An Analysis of the Timing Irregularities for 366 Pulsars. Monthly Notices of the Royal Astronomical Society, 402, 1027-1048. https://doi.org/10.1111/j.1365-2966.2009.15938.x

[15] Cordes, J.M. and Helfand, D.J. (1980) Pulsar Timing. III. Timing Noise of 50 Pulsars. Astrophysical Journal, 239, 640-650. https://doi.org/10.1086/158150

[16] Cordes, J.M. and Shannon, R.M. (2008) Rocking the Lighthouse: Circumpulsar Asteroids and Radio Intermittency. Astrophysical Journal, 682, 1152-1165. https://doi.org/10.1086/589425

[17] Cordes, J.M., Weisberg, J.M. and Boriakoff, V. (1985) Small-Scale Electron Density Turbulence in the Interstellar Medium. Astrophysical Journal, 288, 221-247. https://doi.org/10.1086/162784

[18] D’Alessandro, F., McCulloh, P.M., Hamilton, P.A. AND Deshpande, A.A. (1995) The Timing Noise of 45 Southern Pulsars. Monthly Notices of the Royal Astronomical Society, 277, 1033-1046.

[19] Eya, I.O., Urama, J.O. and Chukwude, A.E. (2019) On the Distributions of Pulsar Glitch Sizes and Inter-Glitch Time Intervals. Research in Astronomy and Astrophysics, 19, 89. https://doi.org/10.1088/1674-4527/19/6/89 
[20] Jones, P.B. (1990) The Generation of Timing Noise by Superfluid Rotation in Pulsars. Monthly Notices of the Royal Astronomical Society, 246, 364-367.

[21] D’Alessandro, F., McCulloch, P.M., Hamilton, P.A. and Deshape, A.A. (1993) Timing Noise of 45 Southern Pulsars. Monthly Notices of the Royal Astronomical Society, 277, 1033-1046.

[22] Cheng, K.S. (1987) Outer Magnetospheric Fluctuations and Pulsar Timing Noise. Astrophysical Journal, 321, 799-804. https://doi.org/10.1086/165672

[23] Kramer, M., Lyne, A.G., O’Brien, J.T., Jordan, C.A. and Lorimer, D.R. (2006) Periodically Active Pulsar Giving Insight into Magnetospheric Physics. Science, 312, 549-551. https://doi.org/10.1126/science. 1124060

[24] Lyne, A.G., Hobbs, G., Kramer, M. and Stairs, B. (2010) Switched Magnetospheric Regulation of Pulsar Spin-Down. Science, 329, 408-412. https://doi.org/10.1126/science.1186683

[25] Chukwude, A.E. and Butcner, S. (2012) Dual Spin-Down States of the Pulsar J1001-5507. Astrophysical Journal, 745, 40-47. https://doi.org/10.1088/0004-637X/745/1/40

[26] Cordes, J.M. and Greenstein, G.S. (1981) Pulsar Timing IV: Physical Models for Timing Noise Models. Astrophysical Journal, 245, 1060-1079. https://doi.org/10.1086/158883

[27] Arzoumanian, Z., Nice, D.J., Taylor, J.H. and Thorsett, S.E. (1994) Timing Behavior of 96 Radio Pulsars. Astrophysical Journal, 422, 671-680. https://doi.org/10.1086/173760

[28] Matsakis, D.N., Taylor, J.H. and Eubanks, T.M. (1997) A Statistic for Describing Pulsar and Clock Stabilities. Astronomy \& Astrophysics, 326, 924-928.

[29] Chukwude, A.E. (2003) On the Statistical Implication of Timing Noise for Pulsar Braking Index. Astronomy \& Astrophysics, 406, 667-671.

https://doi.org/10.1051/0004-6361:20030789

[30] Beskin, G., Biryukov, A. and Karpov, S. (2007) A Note on the Cyclic Evolution of the Pulsar Magnetosphere. Astronomy \& Astrophysics, 475, 639-642.

https://doi.org/10.1051/0004-6361:20078108

[31] Urama, J.O., Link, B. and Weisberg, J.M. (2006) A Strong-Correlation in Radio Pulsars with Implications for Torque Variations. Monthly Notices of the Royal Astronomical Society: Letters, 370, L76-L79.

[32] Birykov, A., Beskin, G. and Karpov, S. (2007) Evidence of Long-Term Cyclic Evolution of Radiopulsar Periods. Advances in Space Research, 40, 1498-1504. https://doi.org/10.1016/j.asr.2007.06.051

[33] Chukwude, A.E. and Odo, F.C. (2016) Assessing the Effects of Timing Irregularities on Radio PulsarsAnomalous Braking Indices. Research in Astronomy and Astrophysics, 16, 150. https://doi.org/10.1088/1674-4527/16/10/150

[34] Chukwude, A.E., Baiden, A.A. and Onuchukwu, C.C. (2010) Measurements of Radio Pulsar Breaking Indices. Astronomy \& Astrophysics, 515, Article No. A21. https://doi.org/10.1051/0004-6361/200911634

[35] Birykov, A., Beskin, G. and Karpov, S. (2012) Monotonic and Cyclic Components of Radio Pulsar Spin-Down. Monthly Notices of the Royal Astronomical Society: Letters, 420, 130-117.

[36] Chukwude, A.E. (2007) A Statistical Analysis of Radio Pulsar Timing Noise. Chinese Journal of Astronomy and Astrophysics, 7, 521-530.

https://doi.org/10.1088/1009-9271/7/4/08 
[37] Iyida, E.U., Eze, C.I., Eya, I.O. and Chukwude, A.E. (2018) On the Effects of Spin Properties on Timing Noise Parameters of Rotation-Powered Pulsars. International Journal of Astrophysics and Space Science, 6, 101-107.

[38] D’Alessandro, F., McCulloch, P.M., King, E.A., Hamilton, P.A. and McConnell, D. (1993) Timing Observations of Southern Pulsars-1987 to 1991. Monthly Notices of the Royal Astronomical Society, 261, 883. 